\title{
NRAS NM_002524.4:C.182A>C
}

National Cancer Institute

\section{Source}

National Cancer Institute. NRAS NM 002524.4:C.182A>C. NCI Thesaurus. Code C98454.

A nucleotide substitution at position 182 of the coding sequence of the NRAS gene

where adenine has been mutated to cytosine. 